\title{
Nano-spin Wave Laser: The Next Generation
}

\section{Yupapin $\mathbf{P P}^{1 *}$, Suwandee $\mathbf{S}^{1}$, Patharacorn $\mathbf{P}^{2}$, Chiangga $\mathbf{S}^{2}$ and Kimura $\mathrm{T}^{3}$}

${ }^{1}$ Faculty of Science and Technology, Interdisciplinary Research Center, Kasem Bundit University, Bangkok 10250, Thailand

${ }^{2}$ Faculty of Science and Technology, Spintronics Research Center, Kasetsart University, Bangkok 10900, Thailand

${ }^{3}$ Department of Physics, Spintronics Research Center, Kyushu University, Fukuoka, Japan

Spin-based electric devices such as giant magneto-resistive (GMR) spin valve and magnetic tunnel junction (MTJ) are also the possible candidate device for highly sensitive magnetic field detection [1-5]. These devices provide significant progress with the developments of spintronics and much better performance compared to other fieldsensing devices such as semiconductor Hall sensor and flux-gate sensor. In addition, magnetic storage, magnetic memory and other functional devices such as semiconductor spin transistor devices and spin quantum bits can be realized by spin-based electric devices. Moreover, recently, the microwave devices based on the dynamical spin motion in sub nano second range have also been demonstrated with highly flexible tenability. Since a few $\mathrm{GHz}$ operations are the physical upper limit of the devices based on the surface acoustic wave which is commonly utilized as the present microwave device, a microwave device based on the spin precession with higher operation frequency is a timely demonstration. Thus, a wide range of applications with various attractive features are expected by utilizing electron spin in the electrical and microwave devices. On the other hand, in each area, the conventional and commercial devices are still improving their device performances. Therefore, the significant developments of the spin devices such as material innovation and novel architecture proposal are essential for overcoming the competition and the wide distribution in the world. In this view point, we believe that the development of the spin device with original functions which are unable to realize in the conventional devices is a key for the breakthrough of the spin devices. To realize this, we are focusing on the spin wave in a panda-shaped nano-ring structure.

Panda ring resonator proposed by us is a high performance semiconductor laser device based on the nonlinear optics (Figure 1). These provide sensitive detection of ultra-small magnetic field comparable to SQUID (superconducting quantum interference device) while its product and running costs, size and structures are much smaller than SQUID. Moreover, by alternating the operation function, it will be possible to extend the optical trapping device such as micro tweezers and virus detector. Thus, Panda ring resonator has various attractive possibilities for human society and healthcare devices. However, unfortunately, an experimental demonstration of the panda ring resonator has not been reported so far although the embedded ring resonator has been demonstrated recently. This is because the integration technology of different-type semiconductors in submicron space is extremely difficult. Our research team has recently found that spin wave in ferromagnetic nanostructures also provides nonlinear operations similar to the semiconductor panda ring resonator because of the analogy between the photon and magnon. So, we combine high performance nonlinear optical and spintronic devices. Namely, instead of optical wave packet, we realize the panda-ring resonator by using spin-wave packet.

In the spin-based devices, periodical magnetization precessions, namely spin wave, can be utilized for the propagation of the spin information. The dynamic property of the spin waves is strongly related to uniformity of the magneto-static interaction. Intrinsically, in nano-structured ferromagnets, the magneto-static interaction becomes inhomogeneous because of the influence of the boundary or the edge. This is important milestone for the miniaturization of the microwave spin device. In this article, we will overcome this serious obstacle by using a ferromagnetic nanoring. Since the influence of the edge/ boundary effects is completely eliminated by the periodical boundary condition of the nanoring, the device performance will be improved significantly. Moreover, by combining the concept of panda resonator developed in the optical laser system, highly attractive novel microwave devices such as spin current amplifier and ultrasensitive magnetic sensor will be developed with the response of the spin wave packet.

In this article, the development of a high performance nano-sized micro/nano scale device called a Panda ring resonator as shown in Figures 1 and 2 [6-8], which is a nonlinear optical/spintronic device for various applications under the concept of nonlinear Panda ring resonator. The ideal operation of the Panda ring resonator will be realized by using the spin-wave packet confined in the coupled ferromagnetic circular nanorings, where the field sensing with nano-meter spatial resolution and ultra-high sensitivity comparable to fabricated device, which will be demonstrated at room temperature with keeping high tenability and flexibility. By extending these sensing techniques, we will also develop the novel high performance spintronic devices such as nanomedical detector and data storage devices and nano-spin wave laser source, which are confirmed by the theoretical works as shown on the publication list. These innovative demonstrations make a breakthrough for spin-based nano-electronic and energy harvesting devices. The spin wave device can be designed, fabricated and tested for various

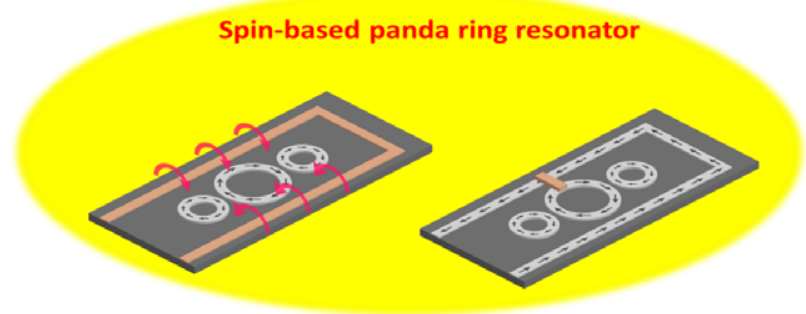

Figure 1: Panda ring resonator structure, where left one is field-driven operation and middle one is spin-current control.

*Corresponding author: Yupapin PP, Faculty of Science and Technology, Interdisciplinary Research Center, Kasem Bundit University, SCI Center, SOL Corporation International Company Limited; Advanced Studies Center, Faculty of Science, King Mongkut's Institute of Technology Ladkrabang, Bangkok 10520, Thailand, Tel: 6623298000; E-mail: kypreech@kmitl.ac.th

Received September 14, 2015; Accepted September 16, 2015; Published September 26, 2015

Citation: Yupapin PP, Suwandee S, Patharacorn P, Chiangga S, Kimura T (2015) Nano-spin Wave Laser: The Next Generation. J Biosens Bioelectron 6: e140. doi:10.4172/2155-6210.1000e140

Copyright: @ 2015 Yupapin PP, et al. This is an open-access article distributed under the terms of the Creative Commons Attribution License, which permits unrestricted use, distribution, and reproduction in any medium, provided the original author and source are credited. 
Citation: Yupapin PP, Suwandee S, Patharacorn P, Chiangga S, Kimura T (2015) Nano-spin Wave Laser: The Next Generation. J Biosens Bioelectron 6: e140. doi:10.4172/2155-6210.1000e140

Page 2 of 2

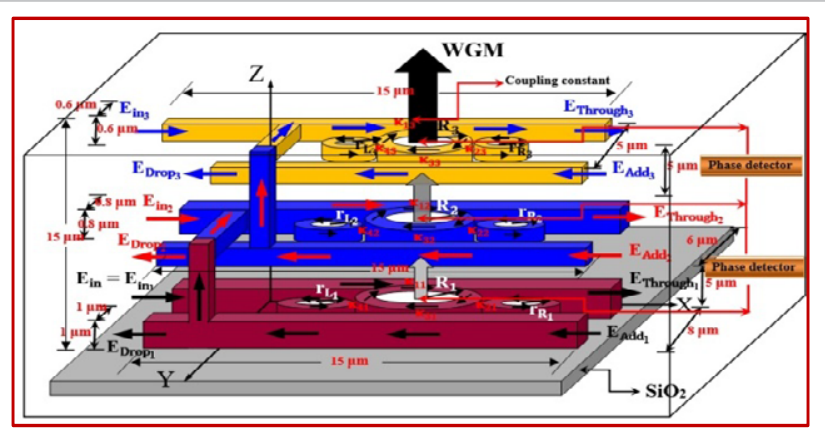

Figure 2: Schematic of nano-laser squeezing system using a Panda ring resonator system, where WGM is the whispering gallery mode output, based on the photon and magnon analogy.

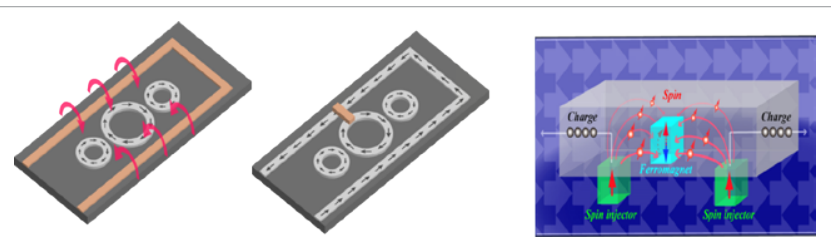

Figure 3: Schematic illustration of spin-based panda ring resonators, where left one is field-driven operation and middle one is spin-current control. Right hand cartoon corresponds to the spin current.

applications. The above device details as shown in Figures 1-3 can be designed and worked similarly for various optical applications, where in this case the metallic device will be used instead of optical device (semiconductor), which can be more reliable used in the fabrication process. The advantage of the nano-spin wave laser is a low power consumption electronic device, where the small scale device within the range of nano-scale device is the other interesting feature.

\section{References}

1. Wolf SA, Awschalom DD, Buhrman RA, Daughton JM, Molnár S, et al. (2001) Spintronics: A spin-based electronics vision for the future, Science 294: 14881495.

2. Kimura T, Hashimoto N, Yamada S, Miyao M, Hamaya K, et al. (2012) Roomtemperature generation of giant pure spin currents using epitaxial $\mathrm{Co}_{2} \mathrm{FeSi}$ spin injectors, NPG (Nature Publishing Group) Asia Mater 4: e13.

3. Kimura T, Otani Y, Hamrle J (2006) Switching magnetization of a nanoscale ferromagnetic particle using nonlocal spin injection. Phys Rev Lett 96: 037201.

4. Mu C, Hu S, Wang J, Kimura T (2013) Thermo-electric effect in a nano-sized crossed Permalloy/Cu junction under high bias current. Appl Phys Lett 103: 132408.

5. Nonoguchi S, Nomura T, Kimura T (2012) Longitudinal and transverse spin current absorptions in a lateral spin valve structure. Phys Rev B 86: 104417.

6. Uomwech K, Sarapat K, Yupapin PP (2010) Dynamic modulated Gaussian pulse propagation within the double PANDA ring resonator system. Microwave and Optical Technology Letters 8: 1818-1821.

7. Phatharaworamet T, Teeka C, Jomtarak R, Mitatha S (2010) Random binary Code generation using dark-bright soliton conversion control within a Panda ring resonator, IEEE Lightwave Technology 19: 2804-2809.

8. Teeka C, Jalil MA, Yupapin PP, Ali J (2010) Novel tunable dynamic tweezers using dark-bright soliton collision control in an optical add/drop filter, IEEE Trans Nanobioscience 4: 258-262. 